\title{
Nivel de ansiedad y depresión por el Coronavirus en gestantes atendidas en establecimientos de salud de Huancayo, 2020
}

Level of anxiety and depression due to Coronavirus in pregnant women treated at health establishments in Huancayo, 2020

\author{
Jenny Mendoza Vilcahuaman ${ }^{1 \text { a, }}$, Cesar Maldonado Gómez ${ }^{1, b}$, Yarid Mauricio Salvatierra ${ }^{1, c}$
}

\section{RESUMEN}

Objetivo: Determinar el nivel de ansiedad y depresión en tiempos de coronavirus de gestantes atendidas en establecimientos de salud de la provincia de Huancayo, 2020. Material y métodos: El tipo de investigación fue observacional, prospectiva, transversal y descriptiva; la muestra fue de 135 gestantes. Resultados: El nivel de ansiedad fue; baja $78,5 \%$, moderada $18,5 \%$ y severa 3,0\%. Nivel de depresión fue, ligera $40,0 \%$, moderada $11,1 \%$, severa $4,5 \%$ y muy severa $0,7 \%$. Las características epidemiológicas fueron; adolescentes $18,5 \%$, jóvenes $61,5 \%$ y adultas $20,0 \%$; convivientes $70,4 \%$, solteras $18,5 \%$; con instrucción primaria $11,1 \%$, secundaria $79,3 \%$ y superior $9,6 \%$; son católicas $68,9 \%$ y cristianas $24,4 \%$. La edad respecto al nivel de ansiedad; las adolescentes tienen ansiedad baja $14,1 \%$ y moderada $4,4 \%$; las jóvenes tienen ansiedad baja $51,1 \%$ y moderada $9,7 \%$; y las adultas tienen ansiedad baja 13,3\%, moderada 4,4\% y severa 2,3\%. La edad respecto al nivel de depresión fue; adolescentes tienen depresión ligera 5,2\% y moderada 1,5\%; las jóvenes tienen depresión ligera $25,9 \%$ y moderada $6,0 \%$; y las adultas tienen depresión ligera $8,9 \%$, moderada $3,7 \%$, severa $3,0 \%$ y muy severa $0,7 \%$. Concluyendo, $100,0 \%$ de las gestantes presentaron ansiedad y el 56,3\% depresión en tiempos de Coronavirus. Conclusiones: Las gestantes con ansiedad baja están con depresión ligera y moderada, las con ansiedad moderada tienen depresión ligera, moderada y severa; y las con ansiedad severa tienen depresión moderada, severa y muy severa.

PALABRAS CLAVE: Gestante, Coronavirus, ansiedad, depresión.

\section{SUMMARY}

The objective of the study was to determine the level of anxiety and depression in times of coronavirus of pregnant women treated in health establishments in Huancayo, 2020. Materials and methods: The type of research was observational, prospective, cross-sectional and descriptive; the sample was 135 pregnant women. Results and conclusion: The anxiety level was; low $78.5 \%$, moderate $18.5 \%$ and severe $3.0 \%$. Depression level was, slight $40.0 \%$, moderate $11.1 \%$, severe $4.5 \%$ and very severe $0.7 \%$. The epidemiological characteristics were; adolescents $18.5 \%$, young people $61.5 \%$ and adults $20.0 \%$; cohabiting $70.4 \%$, single $18.5 \%$; with primary education $11.1 \%$, secondary $79.3 \%$ and higher $9.6 \% ; 68.9 \%$ are Catholic and $24.4 \%$ Christian. The age in relation to the level of anxiety; adolescent girls have low anxiety, $14.1 \%$ and moderate, $4.4 \%$; young women have low anxiety $51.1 \%$ and moderate $9.7 \%$; and the adults have low anxiety $13.3 \%$, moderate $4.4 \%$ and severe $2.3 \%$. The age with respect to

\footnotetext{
${ }^{1}$ Universidad Privada de Huancayo Franklin Roosevelt. Huancayo, Perú.

${ }^{\text {a }}$ Docente; Obstetra; Magister, Doctor. ORCID: 0000-0002-1903-1355

${ }^{\mathrm{b}}$ Docente; Obstetra; Magister. ORCID: 0000-0002-8372-990X

${ }^{c}$ Docente; Obstetra; Magister. ORCID: 0000-0002-2938-4189
} 
the level of depression was; adolescents have mild depression 5.2\% and moderate depression $1.5 \%$; young women have mild depression $25.9 \%$ and moderate $6.0 \%$; and the adults have mild depression $8.9 \%$, moderate $3.7 \%$, severe $3.0 \%$ and very severe $0.7 \%$. In conclusion, $100.0 \%$ of pregnant women presented anxiety and $56.3 \%$ depression in times of Coronavirus; Pregnant women with low anxiety have mild and moderate depression, those with moderate anxiety have mild, moderate and severe depression; and those with severe anxiety have moderate, severe, and very severe depression.

Keywords: Pregnant woman, Coronavirus, anxiety, depression.

\section{INTRODUCCIÓN}

La pandemia del Coronavirus, causada por el COVID-19, ha ocasionado una situación de emergencia mundial que ha puesto en alerta a todos los países y que día a día está cobrando la vida de muchas personas a nivel mundial, no hay distinción de raza, pero si se sabe que los más susceptibles a complicarse son aquellas personas con patologías previas y personas de edades adultos y adultos mayores.

Según la Organización panamericana de la salud (OPS), el 21 de enero del año 2020, el primer caso de COVID 19 se reportó en la región de las Américas, exactamente en el estado de Washington, Estados Unidos de América; unos días después, el 25 de enero, Canadá informó su primer caso confirmado de nuevo coronavirus en Toronto, provincia de Ontario, con esto América del norte tenia al COVID 19 en su población (1).

La pandemia del coronavirus ha cambiado la vida de las personas, y por todo lo que produce en la salud de la persona, se requiere la atención asistencial a una gran cantidad de personas afectadas en su salud física y mental (2).

El Coronavirus, provoca en las personas: miedo, preocupación, zozobra, morbilidad y mortalidad, por ello se asume que esto ocasiona en la gestante mayor ansiedad y depresión de la que se suele encontrarse por la situación propia del embarazo.

La depresión y ansiedad son trastornos afectivos que representan los problemas más frecuentes de la salud mental de la población, en muchos países son una de las primeras causas de discapacidad (3).

La depresión está definida como un trastorno mental que se caracteriza por una profunda tristeza, decaimiento anímico, baja autoestima, pérdida de interés por todo y disminución de las funciones psíquicases (3). Los síntomas clínicos pueden ser; afectivos, somáticos, cognitivos y conductuales, es importante que este trastorno se diagnostique tempranamente, sobre todo en pacientes considerados de riesgo, siendo estos; antecedentes de trastornos afectivos previos, comorbilidades asociadas a la depresión, enfermedades crónicas dolorosas, enfermedades discapacitantes y otros problemas de salud mental (3).

La depresión es más frecuente en las mujeres, se relaciona la etiopatogenia de la depresión con diversos cambios estructurales y bioquímicos que se presentan con la edad, incluye hipofunción serotoninergica, noradrenergica y de la actividad tiroxinhidroxilasa, asi también enfermedades somaticas y perdidas psicológicas que incluyen la soledad, aflicción, y de estatus social $\mathrm{cm}$ la jubilación (3).

La ansiedad es una patología o estado mental que se caracteriza por la presencia de gran inquietud, angustia, una intensa excitación y una extrema inseguridad, y está asociada a hijos de padres ansiosos y sobreprotectores (3).

La persona con ansiedad presenta; sentimientos de temor, actitud de alerta, nerviosismo, inquieto, preocupado, miedo a volverse loco, a morir, la persona es incapaz de relajarse. En estas personas disminuye la atención, se presenta fatiga intelectual, hay mala memoria, alteraciones de sueño, cansancio, sueño no reparador, malhumor, irritabilidad y se encuentra hipervigilante (3).

La ansiedad, en ocasiones se acompaña de la desrealizacion entendida como una situación de extrañeza del entorno y despersonalización donde hay extrañeza e irrealidad personal (3).

Sainz et al., en su estudio Ansiedad y depresión en pacientes embarazadas, estimaron la prevalencia de ansiedad y depresión en pacientes embarazadas de 
diferentes grupos etarios; el método de la investigación fue descriptivo, la muestra 103 embarazadas, y encontraron un promedio de edad de 27,7 años; la escolaridad que predominó fue secundaria 36,9\%, casadas $75,7 \%$, empleadas $62,1 \%$; de forma global se encontró ansiedad en 50,5\% y depresión en $67 \%$; por grupos etarios, la ansiedad se presentó: en el grupo de 12 a 19 años en $41 \%$; en el de 20 a 29 años, $46 \%$; en el de 30 a 34 años, $76 \%$; y de 35 a 39 años, en $46 \%$, con respecto a la depresión en el grupo de 12 a 19 años se observó en $75 \%$; de 20 a 29 años en $74 \%$; de 30 a 34 años en $65 \%$; y de 35 a 39 años en $31 \%$, encontrándose una alta prevalencia de ansiedad y depresión, y mayor prevalencia de depresión en el grupo de 12 a 19 años en comparación con los otros grupos etarios (4).

Díaz et al., en un estudio correlacional, transversal y no experimental, con una muestra de 236 embarazadas excluyendo las con diagnóstico anterior de depresión o ansiedad, enfermedad psiquiátrica previamente diagnosticada, aplicaron los Test de Hamilton para la depresión y ansiedad. $81 \%$ tenía una relación de pareja estable y $54,2 \%$ tenía un embarazo deseado, de las cuales $67,3 \%$ tuvo algún nivel de ansiedad y el $66,1 \%$ algún nivel de depresión (5).

Martínezy Jácome presentaron una revisión de tema acerca de la depresión en el embarazo, sus factores de riesgo, las características clínicas, las complicaciones y el tratamiento (6), en dico estudio utilizaron bases de datos PubMed y LILACS, de ellos 55 cumplían los criterios de inclusión; encontraron que en Sudamérica se registra una prevalencia de aproximadamente el $29 \%$ de depresión, y que los factores de riesgo con mayor significación son el abuso sexual, edad temprana al embarazo y violencia intrafamiliar, concluyendo que el diagnóstico temprano favorece la disminución en las conductas de riesgo, los trastornos del neurodesarrollo fetal y los resultados obstétricos (6).

Lozano e su investigación refiere que, en la lucha contra la epidemia del Coronavirus, el personal de salud experimenta problemas de salud mental tales como estrés, ansiedad, síntomas depresivos, insomnio, negación, ira y temor. La tasa de ansiedad del personal de salud fue del $23,04 \%$, mayor en mujeres que en hombres y mayor entre las enfermeras que entre los médicos, y en la población general de China se observó un $53,8 \%$ de impacto psicológico moderado a severo; $16,5 \%$ de síntomas depresivos, 28,8\% síntomas ansiosos y un $8,1 \%$ de estrés, todos entre moderados y severos (7).
Hoy en día el mundo vive una situación de emergencia en salud, la que esta ocasionada por el virus COVID-19, este virus ha provocado una pandemia que está causando altas tasas de morbilidad y mortalidad en todo grupo poblacional a nivel mundial, y a la fecha está afectando la salud mental de la población en general, Perú no es ajeno a este problema.

El embarazo es una etapa de vida en la mujer que conlleva a una serie de cambios físicos y psicológicos, estos cambios hacen que el estado de la gestación se convierta en un riesgo que puede afectar la salud de las gestantes, sobre todo en el grupo de adolescentes, mujeres con embarazos difíciles y no deseados. Si a esto se le añade la presencia de la pandemia que las expone a un daño físico y emocional, entonces se incrementa el riesgo de daño a su salud no solo mental, sino también física. La presencia del COVID 19 en la población, claramente pone en riesgo la salud mental de las personas y con mayor razón el estado de salud emocional de la gestante.

Los resultados de esta investigación permiten tener un panorama claro de la salud mental de un grupo de gestantes de algunos establecimientos de salud de la provincia de Huancayo, con ello se espera que el personal competente proponga un programa de intervención a favor de la salud mental de las gestantes. El objetivo del presente trabajo fue determinar el nivel de ansiedad y depresión en tiempos de coronavirus de gestantes atendidas en establecimientos de salud de la provincia de Huancayo, 2020.

\section{MATERIAL Y MÉTODOS}

Tipo y diseño de investigación: La investigación fue de tipo; descriptivo porque trabaja con una sola variable, observacional porque no hubo manipulación de la variable, transversal porque se aplicó las escalas en un solo momento y prospectivo (8).

Muestra: Fue de 135 gestantes quienes aceptaron participar en la investigación, dieron su consentimiento informado y fueron entrevistadas, gestantes que se atienden en establecimientos de salud de los distritos de Chilca, Huancayo y El Tambo, de la provincia de Huancayo, meses de octubre a diciembre del 2020. La técnica de muestreo fue no probabilística por conveniencia.

Técnica: Entrevista, aplicada a las gestantes de forma individual, a todas las que asistían a su consulta de atención prenatal. 
Instrumento: Escala de Ansiedad de Beck y escala de Depresión de Hamilton.

Procedimiento: Se pidió permiso a los jefes de los centros de salud de Chilca, La Libertad y Justicia Paz y Vida de la provincia de Huancayo, luego los últimos meses del año 2020 se solicitó el consentimiento informado de cada una de las gestantes que aceptaron participar en la investigación. Los instrumentos fueron las escalas válidas y confiables según Beck y Hamilton, para evaluar el nivel de ansiedad y depresión respectivamente. Las variables fueron codificadas para elaborar la base de datos en Excel.

Técnicas de procesamiento de datos: Se hizo uso de la estadística descriptiva, por ello se elaboraron las tablas de frecuencia a partir de los datos en el programa SPSS versión 23

\section{RESULTADOS}

En la tabla 1 se observa que del 100\% (135 gestantes) que acuden a un establecimiento de salud de la Provincia de Huancayo; No están deprimidos 43,7\% (59), tienen depresión ligera 40,0\% (54 gestantes), depresión moderada $11,1 \%$ (15 gestantes), depresión severa 4,5\% (6 gestantes) y depresión muy severa $0,7 \%$ ( 1 gestante).

En la tabla 2 se observa que del 100\% (135 gestantes) que acuden a un establecimiento de salud de la Provincia de Huancayo; Tienen ansiedad baja $78,5 \%$ (106 gestantes), ansiedad moderada 18,5\% (25 gestantes) y ansiedad severa 3,0\% (4 gestantes).

En la tabla 3 se observa que del 100\% (135 gestantes) que acuden a un establecimiento de salud de la Provincia de Huancayo, tienen; edades de 10 a 19 años $18,5 \%$ ( 25 gestantes), de 20 a 34 años $61,5 \%$ (83

Tabla 1. Nivel de depresión

\begin{tabular}{lccc}
\hline \multicolumn{1}{c}{ Depresión } & $\mathbf{F i}$ & $\mathbf{\%}$ & \% Acumulado \\
\hline No deprimidos & 59 & 43,7 & 43,7 \\
Depresión ligera & 54 & 40,0 & 83,7 \\
Depresión moderada & 15 & 11,1 & 94,8 \\
Depresión severa & 6 & 4,5 & 99,3 \\
Depresión muy severa & 1 & 0,7 & 100,0 \\
Total & 135 & 100,0 & \\
\hline
\end{tabular}

Tabla 2. Nivel de Ansiedad

\begin{tabular}{lccc}
\hline \multicolumn{1}{c}{ Ansiedad } & Fi & \% & \% Acumulado \\
\hline Baja & 106 & 78,5 & 78,5 \\
Moderada & 25 & 18,5 & 97,0 \\
Severa & 4 & 3,0 & 100,0 \\
$\quad$ Total & 135 & 100,0 & \\
\hline
\end{tabular}

Tabla 3. Características epidemiológicas

\begin{tabular}{lcc}
\hline \multicolumn{1}{c}{ Edad } & Fi & \% \\
\hline 10 a 19 años & 25 & 18,5 \\
20 a 34 años & 83 & 61,5 \\
35 a mas años & 27 & 20,0 \\
\multicolumn{1}{c}{ Estado civil } & Fi & $\%$ \\
Conviviente & 95 & 70,4 \\
Soltera & 25 & 18,5 \\
Casada & 15 & 11,1 \\
$\quad$ Grado de instrucción & Fi & $\%$ \\
Primaria & 15 & 11,1 \\
Secundaria & 107 & 79,3 \\
Superior & 13 & 9,6 \\
& Fi & $\%$ \\
Católica & 93 & 68,9 \\
Cristiana & 33 & 24,4 \\
Otros & 9 & 6,7 \\
\hline
\end{tabular}

gestantes) y de 35 a más años 20,0\% (27 gestantes); son convivientes un $70,4 \%$ (95 gestantes), solteras $18,5 \%$ (25 gestantes) y casadas $11,1 \%$ (15 gestantes); tienen instrucción primaria $11,1 \%$ (15 gestantes), secundaria $79,3 \%$ (107 gestantes) y superior $9,6 \%$ (13 gestantes); y son católicas el 68,9\% (93 gestantes), cristianas $24,4 \%$ (33 gestantes) y otras $6,7 \%$ (9 gestantes).

En la tabla 4 se observa que del 100\% (135 gestantes ) que acuden a un establecimiento de salud de la Provincia de Huancayo; las adolescentes tienen ansiedad baja 14,1\% (19 gestantes), ansiedad moderada $4,4 \%$ (6 gestantes); de las jóvenes tienen ansiedad baja 51,1\% (69 gestantes), ansiedad moderada 9,7\% (13 gestantes) y ansiedad severa $0,7 \%$ (1 gestante); y las adultas tienen ansiedad baja 13,3\% (18 gestantes), ansiedad moderada 4,4\% (6 gestantes) y ansiedad severa $2,3 \%$ ( 3 gestantes).

En la tabla 5 se observa que del 100\% (135 gestantes) que acuden a un establecimiento de salud 
Tabla 4. Ansiedad acorde a la edad

\begin{tabular}{lcccccccc}
\hline \multirow{2}{*}{ Edad } & \multicolumn{2}{c}{ Adolescente } & \multicolumn{2}{c}{ Joven } & \multicolumn{2}{c}{ Adulta } & Fi Total & \% \\
\cline { 2 - 7 } Ansiedad & $\mathbf{F i}$ & $\mathbf{\%}$ & $\mathbf{F i}$ & $\mathbf{\%}$ & $\mathbf{F i}$ & $\mathbf{\%}$ & & \\
\hline Baja & 19 & 14,1 & 69 & 51,1 & 18 & 13,3 & 106 & 78,5 \\
Moderada & 6 & 4,4 & 13 & 9,7 & 6 & 4,4 & 25 & 18,5 \\
Severa & 0 & 0,0 & 1 & 0,7 & 3 & 2,3 & 4 & 3,0 \\
Total & 25 & 18,5 & 83 & 61,5 & 27 & 20,0 & 3,0 & 100,0 \\
\hline
\end{tabular}

Tabla 5. Depresión acorde a la edad

\begin{tabular}{lcccccccc}
\hline \multirow{2}{*}{ Edad } & \multicolumn{2}{c}{ Adolescente } & \multicolumn{2}{c}{ Joven } & \multicolumn{2}{c}{ Adulta } & Fi Total & \% \\
\cline { 2 - 7 } & $\mathbf{F i}$ & $\mathbf{\%}$ & $\mathbf{F i}$ & $\mathbf{\%}$ & $\mathbf{F i}$ & $\mathbf{\%}$ & & \\
\hline No deprimidos & 15 & 11,1 & 39 & 28,9 & 5 & 3,7 & 59 & 43,7 \\
Ligera & 7 & 5,2 & 35 & 25,9 & 12 & 8,9 & 54 & 40,0 \\
Moderada & 2 & 1,5 & 8 & 6,0 & 5 & 3,7 & 15 & 11,1 \\
Severa & 1 & 0,7 & 1 & 0,7 & 4 & 3,0 & 6 & 4,5 \\
Muy severa & 0 & 0,0 & 0 & 0,0 & 1 & 0,7 & 1 & 0,7 \\
Total & 25 & 18,5 & 83 & 61,5 & 27 & 20,0 & 3,0 & 100,0 \\
\hline
\end{tabular}

Tabla 6. Ansiedad y depresión

\begin{tabular}{lcccccccc}
\hline \multirow{2}{*}{$\begin{array}{c}\text { Ansiedad } \\
\text { Depresión }\end{array}$} & \multicolumn{2}{c}{ Baja } & \multicolumn{7}{c}{ Moderada } & \multicolumn{2}{c}{ Severa } & Fi Total & \% \\
\cline { 2 - 7 } & Fi & \% & Fi & \% & Fi & \% & & \\
\hline No deprimidos & 51 & 37,8 & 8 & 6,0 & 0 & 0,0 & 59 & 43,7 \\
Ligera & 46 & 34,1 & 7 & 5,1 & 1 & 0,7 & 54 & 40,0 \\
Moderada & 8 & 6,0 & 6 & 4,4 & 1 & 0,8 & 15 & 11,1 \\
Severa & 1 & 0,7 & 4 & 3,0 & 1 & 0,8 & 6 & 4,5 \\
Muy severa & 0 & 0,0 & 0 & 0,0 & 1 & 0,7 & 1 & 0,7 \\
Total & 106 & 78,5 & 25 & 18,5 & 4 & 3,0 & 3,0 & 100,0 \\
\hline
\end{tabular}

de la Provincia de Huancayo; Las adolescentes tienen depresión ligera 5,2\% (7 gestantes), moderada 1,5\% ( 2 gestantes), severa $0,7 \%$ ( 1 gestante) y no están deprimidas un $11,1 \%$ (15); de las jóvenes tienen depresión ligera $25,9 \%$ (35 gestantes), moderada $6,0 \%$ (8 gestantes), severa $0,7 \%$ ( 1 gestante $)$ y no tienen depresión 28,9\% (39 gestantes); y de las adultas tienen depresión ligera 8,9\% (12 gestantes), moderada 3,7\%
(5 gestantes), severa 3,0 (4 gestantes) y muy severa $0,7 \%$ ( 1 gestante) y no están deprimidas $3,7 \%$ (5 gestantes).

En la tabla 6 se observa que del 100\% (135 gestantes) que acuden a un establecimiento de salud de la Provincia de Huancayo; De las gestantes con ansiedad baja están con depresión ligera 34,1\% (46 
gestantes), moderada $6,0 \%$ ( 8 gestantes), severa $0,7 \%$ (1 gestantes) y sin depresión $37,8 \%$ (51 gestantes); De las gestantes con ansiedad moderada tienen depresión ligera 5,1\% (7 gestantes), moderada 4,4\% (6 gestantes), severa 3,0\% (4 gestantes) y sin depresión $6,0 \%$ ( 8 gestantes); y de las gestantes con ansiedad severa tienen depresión ligera $0,7 \%$ (1 gestante), moderada $0,8 \%$ ( 1 gestante), severa $0,8 \%$ (1 gestante) y muy severa $0,7 \%$ ( 1 gestante).

\section{DISCUSIÓN}

El nivel de ansiedad por el coronavirus en gestantes atendidas en establecimientos de salud de la provincia de Huancayo, tienen ansiedad baja 78,5\% (106), ansiedad moderada $18,5 \%$ (25 gestantes) y ansiedad severa 3,0\% (4 gestantes). No están deprimidos 43,7\% (59 gestantes); estos resultados no concuerdan con los reportados por Sainz et al., quienes hallaron en su muestra de estudio que la ansiedad se encontró en el 50,5\% (4); ni con los de con los estudios de Díaz et al (5), en su estudio 67,3\% tuvo algún nivel de ansiedad. Estos nos indica de manera puntual la ansiedad es frecuente, sin embargo, por estar viviendo una situación de emergencia en salud por acusa del COVID 19, el 100\% de las gestantes de la provincia de Huancayo tienen ansiedad en algún nivel.

En tiempos del Coronavirus, el 56,3\% de gestantes atendidas en establecimientos de salud de la provincia de Huancayo presentaron depresión, y de ellas, tienen depresión ligera 40,0\% (54), depresión moderada $11,1 \%$ (15 gestantes), depresión severa 4,5\% (6 gestantes) y depresión muy severa $0,7 \%$ (1 gestantes). Estos resultados concuerdan con los reportados por Sainz et al., quienes reportaron en su muestra de estudio que la depresión estuvo presente en el 67\% de su muestra (4); y con los estudios de Díaz et al., en su estudio el $66,1 \%$ tenía algún nivel de depresión (5), estos resultados nos indican que la depresión es un trastorno mental frecuente en las gestantes, y que por ello es importante mantener un trabajo interdisciplinario con los psicólogos y psiquiatras de ser necesario durante el embarazo.

Esta investigación encontró que, el nivel de ansiedad y depresión por el coronavirus en gestantes atendidas en establecimientos de salud de la provincia de Huancayo, del 100\% (135 gestantes); De las gestantes con ansiedad baja están con depresión ligera $34,1 \%$ (46 gestantes), moderada $6,0 \%$ (8 gestantes), severa $0,7 \%$ (1 gestante) y sin depresión $37,8 \%$ (51 gestantes); De las gestantes con ansiedad moderada tienen depresión ligera 5,1\% (7 gestantes), moderada $4,4 \%$ (6 gestantes), severa $3,0 \%$ (4 gestantes) y $\sin$ depresión $6,0 \%$ (8 gestantes); y de las gestantes con ansiedad severa tienen depresión ligera $0,7 \%$ (1), moderada $0,8 \%$ ( 1 gestante), severa $0,8 \%$ ( 1 gestante) y muy severa $0,7 \%$ ( 1 gestante). Esto nos indica que el $100 \%$ de las gestantes con ansiedad severa, están deprimidas. Es importante en estos tiempos del Coronavirus fortalecer la atención de la salud mental de las gestantes de la provincia de Huancayo.

Las características epidemiológicas de las gestantes con ansiedad y depresión por el coronavirus en gestantes atendidas en establecimientos de salud de la provincia de Huancayo; tienen de 10 a 19 años $18,5 \%$ (25 gestantes), de 20 a 34 años $61,5 \%$ (8 gestantes 3 ) y de 35 a más años $20,0 \%$ (27 gestantes); convivientes un $70,4 \%$ (95 gestantes), solteras $18,5 \%$ (25 gestantes) y casadas $11,1 \%$ (15 gestantes); tienen instrucción primaria $11,1 \%$ (15 gestantes), secundaria 79,3\% (107 gestantes) y superior 9,6\% (13 gestantes) y son católicas el 68,9\% (93 gestantes) y cristianas 24,4\% (33 gestantes). Según Martínez y Jácome, en Sudamérica se registra una prevalencia de aproximadamente el $29 \%$ de depresión en gestantes, y son más frecuentes en edades tempranas (6), lo que hace concluir que las gestantes jóvenes son más propensas a presentar trastornes mentales como ansiedad y depresión.

La características edad con respecto al nivel de ansiedad de las gestantes atendidas, del 100\% (135 gestantes); Las adolescentes tienen ansiedad baja 14,1\% (19 gestantes), ansiedad moderada 4,4\% (6 gestantes); de las jóvenes tienen ansiedad baja 51,1\% (69 gestantes), ansiedad moderada 9,7\% (13 gestantes) y ansiedad severa $0,7 \%$ (1 gestante); y las adultas tienen ansiedad baja 13,3\% (18 gestantes), ansiedad moderada 4,4\% (6 gestantes) y ansiedad severa 2,3\% (3 gestantes), estos resultados indican que las gestantes adultas de la provincia de Huancayo, son más ansiosas que las jóvenes.

La características edad con respecto al nivel de depresión de las gestantes atendidas, se observa que del 100\% (135 gestantes); Las adolescentes tienen depresión ligera 5,2\% (7 gestantes), moderada 1,5\% (2 gestantes), severa 0,7\% (1); de las jóvenes tienen depresión ligera $25,9 \%$ (35 gestantes), moderada $6,0 \%$ ( 8 gestantes), severa $0,7 \%$ ( 1 gestante); y de las adultas tienen depresión ligera 8,9\% (12 gestantes), moderada 3,7\% (5 gestantes), severa 3,0 (4 gestantes) y muy severa $0,7 \%$ ( 1 gestante). Aquí también se observa que las gestantes adultas son las que más se 
deprimen, este es un interesante resultado ya que la edad en la que se hubiera esperado haya más depresión es la adolescencia, por todo lo que implica ser gestante adolescente y vivir en situación de emergencia causada por el Coronavirus.

\section{CONCLUSIONES}

El nivel de ansiedad y depresión por el coronavirus en gestantes atendidas en establecimientos de salud de la provincia de Huancayo, muestran; gestantes con ansiedad baja están con depresión ligera 34,1\%, moderada $6,0 \%$, severa $0,7 \%$ y sin depresión $37,8 \%$; De las gestantes con ansiedad moderada tienen depresión ligera $5,1 \%$, moderada $4,4 \%$, severa $3,0 \%$ y sin depresión $6,0 \%$; y de las gestantes con ansiedad severa tienen depresión ligera $0,7 \%$, moderada $0,8 \%$, severa $0,8 \%$ y muy severa $0,7 \%$.

El nivel de ansiedad por el coronavirus en gestantes atendidas en establecimientos de salud de la provincia de Huancayo, indican; ansiedad baja 78,5\%, ansiedad moderada $18,5 \%$ y ansiedad severa $3,0 \%$.

El nivel de depresión por el coronavirus en gestantes atendidas en establecimientos de salud de la provincia de Huancayo, tienen; depresión ligera $40,0 \%$, depresión moderada $11,1 \%$, depresión severa $4,5 \%$ y depresión muy severa $0,7 \%$.

Las características epidemiológicas de las gestantes con ansiedad y depresión por el coronavirus fueron; son adolescentes 18,5\%, jóvenes $61,5 \%$ y adultas $20,0 \%$; convivientes un $70,4 \%$, solteras $18,5 \%$ y casadas $11,1 \%$; con instrucción primaria $11,1 \%$, secundaria $79,3 \%$ y superior $9,6 \%$ y son católicas el $68,9 \%$ y cristianas 24,4 .

La características edad con respecto al nivel de ansiedad de las gestantes atendidas, muestran; Las adolescentes tienen ansiedad baja $14,1 \%$, ansiedad moderada $4,4 \%$; de las jóvenes tienen ansiedad baja $51,1 \%$, ansiedad moderada $9,7 \%$ y ansiedad severa $0,7 \%$; y las adultas tienen ansiedad baja $13,3 \%$, ansiedad moderada $4,4 \%$ y ansiedad severa $2,3 \%$.

La características de edad con respecto al nivel de depresión de las gestantes atendidas, se observa que del $100 \%$, las adolescentes tienen depresión ligera $5,2 \%$, moderada $1,5 \%$, severa $0,7 \%$; de las jóvenes tienen depresión ligera $25,9 \%$, moderada $6,0 \%$, severa $0,7 \%$; y de las adultas tienen depresión ligera $8,9 \%$, moderada $3,7 \%$, severa 3,0 y muy severa $0,7 \%$.
Agradecimiento:Gracias a la Universidad Privada de Huancayo Franklin Roosevelt por haber financiado esta investigación. Gracias a cada una de las gestantes que aceptaron participar en nuestra investigación.

\section{Correspondencia}

Jenny Mendoza Vilcahuaman

Correo electrónico:jmendoza@uroosevelt.edu.pe

Fecha de recepción: 20 de enero del 2021

Fecha de aceptación: 10 de junio del 2021

\section{REFERENCIAS BIBLIOGRÁFICAS}

1. Organización Panamericana de la Salud. Actualización Epidemiológica: Nuevo coronavirus (2019-nCoV) al 27 de enero de 2020, Washington DC: Organización Panamericana de la Salud; 2020.

2. Ministerio de Salud. Cuidado de la salud mental del personal de la salud en el contexto del COVID-19. Lima, Perú: Ministerio de Salud; 2020. (Citado el 29 de abril del 2020). Disponible en: http://bvs.minsa. gob.pe/local/MINSA/5000.pdf

3. Goncalves F, Gonzales V, Vásquez J. Guía de buena práctica clínica en depresión y ansiedad.. Madrid, Españ: Organización Médica Colegial de España; 2008. (Citado el 29 de abril del 2020). Disponible en: https://www.cgcom.es/sites/default/ files/guia_depresion_ansiedad_2_edicion.pdf

4. Sainz K, Chavez B, Díaz M, Sandoval M, Robles M. Ansiedad y depresión en pacientes embarazadas. Rev Atención familiar. 2013; 20(1): 25-27. (Citado el 29 de abril del 2020). Disponible en: https://www.sciencedirect.com/science/article/pii/ S1405887116300827

5. Díaz M, Amato R, Chavez J, Ramirez M, Rangel S, Rivera L, Lopez J. Depresion y ansiedad en embarazadas. Salus. 2013; 17(2). (Citado el 29 de abril del 2020). Disponible en: http:// ve.scielo.org/scielo.php?script=sci_arttext\&pid $=$ S1316-71382013000200006

6. Martínez P, Jácome N. Depresión en el embarazo. Rev Colomb Psiquiat. 2019 ;48(1): 58-65. (Citado el 29 de abril del 2020). Disponible en: http://www. scielo.org.co/pdf/rcp/v48n1/0034-7450rcp-48-01-58.pdf

7. Lozano VA. Impacto de la epidemia del Coronavirus (COVID-19) en la salud mental del personal de salud y en la población general de China. Rev Neuropsiquiatr. 2020; 83(1):51-56.

8. Supo J. Metodología de la investigación científica. Lima: Sociedad Hispana de investigadores Cientificos;2020.

9. Beck AT, Steer R. Beck Anxiety Inventory manual. San Antonio, TX: Psychological Corporation; 1993. 
10. Sanz J, Garcia-Vera M, Fortún M. El "Inventario de ansiedad de Beck" (BAI): propiedades psicométricas de la versión española en pacientes con trastornos psicológicos. Psicología Conductual 2012;20 (3): 563-583. (Citado el 29 de abril del 2020). Disponible en: https:/www.behavioralpsycho.com/wp-content/ uploads/2019/08/05.Sanz_20-3oa.pdf

11. Hamilton, M., Development of a rating scale for primary depressive illness. Br J Soc Clin Psychol. 1967;6: 278-296.

12. Purriños M. Escala de Hamilton. Galicia: Medicina Interna de Galicia; 20190. (Citado el 29 de abril del 2020). Disponible en: https://meiga.info/escalas/ depresion-escala-hamilton.PDF
13. National Institute for Clinical Excellence. Depression: management of depression in primary and secondary care- NICE guidance. Londres: National Institute for Clinical Excellence; 2004 (Citado el 29 de abril del 2020). Disponible en: https://www.nice.org.uk/ guidance/cg23/documents/cg23-depression-niceguideline-marked-up-with-proposed-amendments2

Recibido: $15 / 12 / 2020$

Aceptado: 14/04/2021 